\title{
Using tyrosinase as a tri-modality reporter gene to monitor transplanted stem cells in acute myocardial infarction
}

Mei Liu ${ }^{1,2}$, Yichun Wang ${ }^{1,2}$, Mengting Li ${ }^{1,2}$, Hongyan Feng ${ }^{1,2}$, Qingyao Liu ${ }^{1,2}$, Chunxia Qin ${ }^{1,2}$, Yongxue Zhang ${ }^{1,2}$ and Xiaoli Lan (1) ${ }^{1,2}$

\begin{abstract}
The study aimed to investigate the feasibility of noninvasive monitoring of bone marrow mesenchymal stem cells (MSCs) transduced with the tyrosinase reporter gene for acute myocardial infarction (AMI) with photoacoustic imaging (PAI), magnetic resonance imaging (MRI), and positron emission tomography (PET) in vitro and in vivo. MSCs were transduced with a lentivirus carrying a tyrosinase reporter gene. After transduction, the rate of ${ }^{18} \mathrm{~F}-5$-fluoro- $\mathrm{N}-(2-$ [diethylamino]ethyl)picolinamide $\left({ }^{18} \mathrm{~F}-5-\mathrm{FPN}\right)$ uptake was measured. PAI and MRI of stable cell lines expressing tyrosinase (TYR-MSCs) were performed in vitro. An AMI model was induced and verified. TYR-MSCs and MSCs were injected into the margins of the infarcted areas, and PAI, MRI, and PET images were acquired 1, 7, 14, 21, and 28 days after cell injection. Sham-operated models without injection were used as the control group. TYR-MSCs showed noticeably higher uptake of ${ }^{18} \mathrm{~F}$-5-FPN and stronger signals in T1-weighted MRI and PAI than non-transduced MSCs. In vivo studies revealed prominent signals in the injected area of the infarcted myocardium on PAI/MRI/PET images, whereas no signal could be seen in rats injected with non-transduced MSCs or sham-operated rats. The uptake values of ${ }^{18} \mathrm{~F}-5$-FPN in vivo showed a slight decrease over 28 days, whereas MRI and PAI signal intensity decreased dramatically. MSCs stably transduced with the tyrosinase reporter gene could be monitored in vivo in myocardial infarction models by PET, MRI, and PAl, providing a feasible and reliable method for checking the viability, location, and dwell time of transplanted stem cells.
\end{abstract}

\section{Introduction}

Stem cell transplantation is a new method aimed at reversing myocardial injury and improving cardiac function $^{1}$. Unfortunately, only a minute number of these laboratory studies can be transferred to clinical practice due to limited methods to monitor the fate of bone marrow mesenchymal stem cells (MSCs) after their transplantation into injured hearts. Recent developments in imaging systems and new probes have allowed investigators to perform

\footnotetext{
Correspondence: Xiaoli Lan (LXL730724@hotmail.com)

'Department of Nuclear Medicine, Union Hospital, Tongji Medical College,

Huazhong University of Science and Technology, 430022 Wuhan, China

${ }^{2}$ Hubei Province Key Laboratory of Molecular Imaging, Union Hospital, Tongji

Medical College, Huazhong University of Science and Technology, 430022

Wuhan, China
}

multimodality imaging in animal models that have employed MSC transplantation. For example, Pei et al. ${ }^{2}$ constructed a triple-fused reporter gene that combined herpes simplex virus type 1 thymidine kinase, enhanced green fluorescent protein, and firefly luciferase to monitor stem cells using positron emission tomography (PET), fluorescence, and bioluminescence imaging. However, integrating different active groups into one entity is complicated and time-consuming. Most importantly, the deactivation of one functional group will lead to failure of the whole construction. In addition, the employed adenovirus transduction leads to transient gene expression, and gene expression levels decline quickly with time. It is necessary to obtain a simple multimodality molecular probe that is easily constructed and stably expressed. 
Human tyrosinase, the key enzyme in melanin production, was first evaluated by Qin et al. ${ }^{3}$ as a stand-alone reporter gene for in vitro and in vivo multimodality imaging, including photoacoustic imaging (PAI), magnetic resonance imaging (MRI), and PET. We first established a tyrosinase reporter gene system under the control of the Tet-on gene expression system in vitro in tumor cell lines, which were proof-of-concept studies ${ }^{4}$. In this study, tyrosinase was used as a multifunctional reporter gene to track stem cells transplanted in an area of myocardial infarction. After transducing the tyrosinase gene into MSCs in Sprague-Dawley (SD) rats, melanin was synthesized and subsequently absorbed light energy to achieve $\mathrm{PAI}^{5}$, bound with ${ }^{18} \mathrm{~F}$-5-fluoro- $N$-(2-[diethylamino]ethyl)picolinamide $\left({ }^{18} \mathrm{~F}-5\right.$-FPN $)$ specifically to enable PET imaging ${ }^{6}$, and combined with iron for visibility on $\mathrm{MRI}^{7}$. The aim of this study was to establish a simple multimodality reporter gene system using tyrosinase and combining PAI, PET, and MRI to monitor the fate of MSCs after transplantation into rat models of AMI.

\section{Materials and Methods \\ PET imaging tracers}

${ }^{18} \mathrm{~F}-5$-FPN is a benzamide analog that specifically binds melanin. The synthesis process was performed according to our previously published procedures ${ }^{6}$. The reaction was completed in a synthesis module (GE TraceLab FX-XN, GE Healthcare, Milwaukee, WI, USA).

\section{Construction of a recombinant lentiviral vector carrying the tyrosinase reporter gene}

Tyrosinase complementary DNA was kindly provided by Dr. Zhen Cheng of Stanford University. The tyrosinase sequence was amplified and digested with AgeI/NheI, then cloned into the Ubi-MCS-3FLAG-SV40-puromycin vector, which carried puromycin-resistance genes (Shanghai Genechem Co. Ltd., Shanghai, China).

\section{Isolation, cultivation, and identification of TYR-MSCs}

All experiments were performed in accordance with protocols approved by the Animal Care and Use Committee of Huazhong University of Science and Technology, China. Rat bone marrow MSCs were isolated and purified from 4-week-old SD rats (male, $80-100 \mathrm{~g}$ ) by combing gradient density centrifugation and adhesion separation. The surface makers CD44, CD90, CD34, and CD33 (antibodies from Abcam, Cambridge, MA, USA) were detected by flow cytometry. Transduction of tyrosinase lentiviral vectors into MSCs was performed at a multiplicity of infection of $3^{8}$. Antibiotic selection with $1.75 \mu \mathrm{g} / \mathrm{mL}$ puromycin began $72 \mathrm{~h}$ after transfection and lasted for 5 days to remove the MSCs that were not transduced successfully 9 . Masson-Fontana, western blot, and tyrosinase activity assays were performed to demonstrate enhanced melanin synthesis in stably transduced cell clones ${ }^{10}$.

\section{In vitro cellular uptake assays}

Cellular uptake assays were performed on TYR-MSCs, MSCs, and TYR-MSCs + blocking. Cells were seeded in 24-well plates at a density of $2 \times 10^{5}$ cells per well. After overnight incubation, cells were washed twice with PBS. Then, $200 \mu \mathrm{L}$ of completed DMEM-F 12 medium containing $37 \mathrm{kBq}$ of ${ }^{18} \mathrm{~F}-5$-FPN was added to each well. After incubation with ${ }^{18} \mathrm{~F}-5$-FPN at $37^{\circ} \mathrm{C}$ for increasing intervals (30,60, and $120 \mathrm{~min})$, media were removed, and cells were washed twice with PBS and lysed with $1 \mathrm{~N} \mathrm{NaOH}$ for $5 \mathrm{~min}$. For the blocking study, TYR-MSCs were incubated for $1 \mathrm{~h}$ at $37^{\circ} \mathrm{C}$ with ${ }^{18} \mathrm{~F}-5$-FPN (37 kBq) in the presence of $100 \mu \mathrm{L}$ of $10^{-5} \mathrm{M}$ standard ${ }^{19} \mathrm{~F}-5$-FPN. Radioactivity was measured using a gamma counter (2470, WIZARD; PerkinElmer, Waltham, MA, USA). The uptake rate was obtained using the following equation ${ }^{2}$, and all the above experiments were performed three times with triplicate wells.

$$
\begin{gathered}
\text { Uptake rate }(\%)= \\
{\left[\text { counts }_{\text {intracellular }} /\left(\text { counts }_{\text {extracellular }+ \text { counts }} \text { intracellular }\right)\right] \times 100 \%}
\end{gathered}
$$

\section{In vitro MRI}

MRI of cells was performed using a 7.0 T MRI (Varian, Palo Alto, CA, USA). Increasing numbers of cells $\left(5 \times 10^{5}\right.$, $2.5 \times 10^{6}$, and $5 \times 10^{6}$ ) were embedded in $1 \%$ agarose $^{11}$, and T1-weighted imaging (T1WI) was performed ${ }^{12}$. To increase the production of melanin, the TYR-MSCs + tyrosine line was pretreated with $2 \mathrm{mM}$ tyrosine for $24 \mathrm{~h}$. T1WI parameters included a field of view $7.0 \times 3.5 \mathrm{~cm}$, matrix size of $256 \times 256$, section thickness of $1 \mathrm{~mm}$, repetition time of $500 \mathrm{~ms}$, and echo time of $11 \mathrm{~ms}$. Image analysis was performed using Image $(\mathrm{NIH}$, Bethesda, MD, USA). The experiments were performed three times with triplicate wells.

\section{In vitro PAI}

PAI (Endra Nexus 128 Photoacoustic Imaging System, Endra Life Sciences, Ann Arbor, MI, USA) of increasing concentrations of cells, ranging from $5 \times 10^{4}$ to $1 \times 10^{7}$ cells per $\mathrm{mL}$ and embedded in $1 \%$ agarose, was performed. Photoacoustic signals generated by a given laser pulse in a target at a 5-mm depth and 680-nm wavelength in a phantom were detected by all transducers ${ }^{13}$. Data were reconstructed and drawn in Osirix after exporting the raw data in DICOM format (Pixmeo, Switzerland). The experiments were performed three times with triplicate wells.

\section{Acute myocardial infarction animal model preparation}

Adult SD rats (male, 160-200 g) were anesthetized by an intraperitoneal (ip) injection of $3 \%$ sodium 
pentobarbital ( $35 \mathrm{mg} / \mathrm{kg}$, Merck, Darmstadt, Germany) and artificially ventilated with an animal ventilator (DW3000, Jinyang Wanda, Beijing, China). Left thoracotomy was performed, and the left anterior descending coronary artery was permanently ligated at its origin to establish acute myocardial infarction (AMI) models ${ }^{14}$. The shamoperated controls underwent the same surgery except that the coronary artery was not ligated. A total of 21 AMI models and 13 control models were successfully established and used for in vivo experiments. Thirty minutes after ligation of the left anterior descending coronary artery, eight injections of TYR-MSCs or MSCs $\left(2 \times 10^{6}\right.$ cells per $50 \mu \mathrm{L}$ PBS) were injected into the margin of the infarcted area. Seventy-two hours after ligation of the coronary artery, five other AMI models without transplanted TYR-MSCs or MSCs were humanely killed to perform 2,3,5-Triphenyltetrazolium chloride (TTC) $(n=$ $3)$ or hematoxylin and eosin staining $(n=2)$. Five control models were also killed to perform TTC $(n=3)$ or hematoxylin and eosin staining $(n=2)$. Infarct size was expressed as a percentage of total left ventricular area. The mean of all slices from each heart was calculated. Left ventricular (LV) infarct size was measured in six to eight transverse sections of 1-2 mm from apex to base stain. The infract zone was identified by a white color. According to the result of TTC staining, infarction size was calculated using the following formula: ${ }^{2}$

$$
\begin{aligned}
& \text { Infarct size }(\%)= \\
& (\text { infarcted area } / \text { total left ventricle area }) \times 100 \% .
\end{aligned}
$$

To perform hematoxylin and eosin staining, the hearts of the models were quickly removed and cut into six transverse slices from apex to base. Subsequently, partial $8-\mu \mathrm{m}$ transverse slices from each section were prepared for hematoxylin and eosin staining.

\footnotetext{
${ }^{18} \mathrm{~F}$-FDG myocardial metabolic imaging was performed to confirm successful coronary occlusion in TYR-MSCs $(n=8)$, MSCs $(n=8)$ transplanted AMI models $24 \mathrm{~h}$ before ${ }^{18} \mathrm{~F}-5$-FPN imaging ${ }^{15}$. MRI, PET, and PAI were performed at five points post operation: $1,7,14,21$, and 28 days. Control models $(n=8)$ were also subjected to ${ }^{18} \mathrm{~F}$ 5-FPN imaging, MRI, PET, and PAI at the same time points.
}

\section{In vivo ${ }^{18} \mathrm{~F}$-FDG myocardial metabolic imaging}

After fasting for $4 \mathrm{~h}, 16 \mathrm{AMI}$ rats, 8 control models, and 2 normal rats were anesthetized with $2 \%$ isoflurane in oxygen and injected via the tail vein with a mean dose of 3.7 MBq of ${ }^{18}$ F-FDG. A PET scan (Trans-PET BioCaliburn 700, Raycan Technology Co., Ltd., Suzhou, China) focused on the chest was acquired for 15 min starting 60 min after the injection.

\section{In vivo ${ }^{18} \mathrm{~F}-5$-FPN animal $\mathrm{PET}$ imaging}

The TYR-MSCs group $(n=8)$, MSCs group $(n=8)$, and control group $(n=8)$ were injected intravenously with $3.7 \mathrm{MBq}$ of ${ }^{18} \mathrm{~F}-5$-FPN. After $1 \mathrm{~h}$, rats were anesthetized with $2 \%$ isoflurane in oxygen and placed in the prone position. The PET scan acquisition conditions were identical to those with ${ }^{18} \mathrm{~F}$-FDG. This algorithm produces images consisting of $0.5 \times 0.5 \times 0.5 \mathrm{~mm}^{3}$ voxels. Images were reconstructed into a $280 \times 280 \times 104$ matrix using 3D OSEM with a pixel size of $0.5 \times 0.5 \mathrm{~mm}^{2}$ and a slice thickness of $0.5 \mathrm{~mm}$. Regions of interest (ROIs) were marked manually and analyzed by AMIDE (UCLA, Los Angeles, CA, USA). Signals were expressed as a percent of injected dose per gram of tissue (\%ID per g).

\section{In vivo MRI imaging}

The TYR-MSCs group $(n=8)$, MSCs group $(n=8)$, and control group $(n=8)$ were continuously anesthetized with $2 \%$ isoflurane in oxygen and placed in the prone position. The scanning parameters were as follows: repetition time of $48 \mathrm{~ms}$; echo time of $3 \mathrm{~ms}$; slice thickness of $1 \mathrm{~mm}$; field of view of $4.5 \times 4.5 \mathrm{~cm}$; and $192 \times 192$ matrix. T2*-weighted images were detected as hypointensities caused by $\mathrm{Fe}^{3+}$-bound melanin ${ }^{16}$. Image analysis was performed using ImageJ.

\section{In vivo $\mathrm{PAl}$ imaging}

The TYR-MSCs group $(n=8)$, MSCs group $(n=8)$, and control group $(n=8)$ were anesthetized via ip injection of $3 \%$ sodium pentobarbital $(35 \mathrm{mg} / \mathrm{kg})$ using the same PAI system employed for the in vitro studies and the same wavelength ${ }^{17}$. After scanning a rat, signals were sent to ultrasonic transducers and then directed to a computer system to reconstruct two-dimensional and three-dimensional images using imaging software (OsiriX Foundation, Geneva, Switzerland) ${ }^{18}$.

\section{Statistical analysis}

Quantitative data are expressed as the means \pm SD. Statistical analysis was performed using one-way analysis of variance and the Student's $t$ test (SPSS 16.0 software package, SPSS Inc., Chicago, IL, USA). A 95\% confidence interval was chosen to determine the significance of differences between groups. A probability value of $P<0.05$ was considered statistically significant.

\section{Results}

\section{In vitro tyrosinase introduction and melanin expression in} MSCs

MSCs were successfully transduced with lentivirus carrying the tyrosinase reporter gene and selected with puromycin. After centrifugation, TYR-MSCs were black in color, whereas non-transduced MSCs were white or light yellow (Fig. 1a). TYR-MSCs showed a specific band 
A

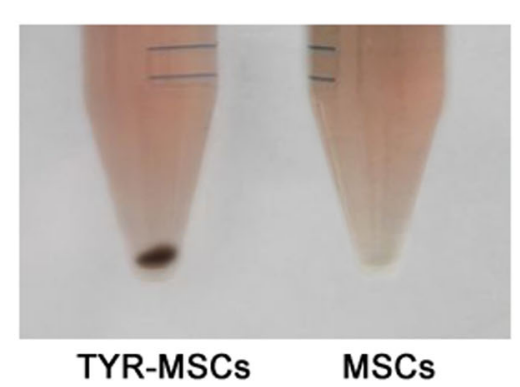

B

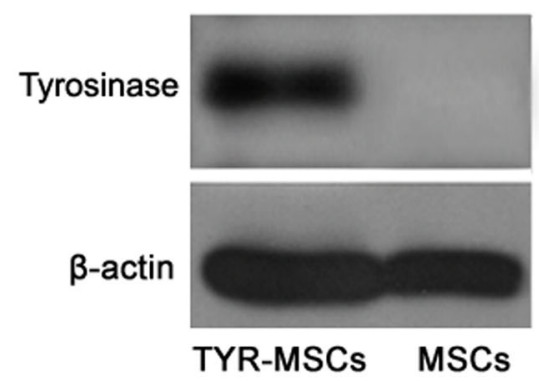

C
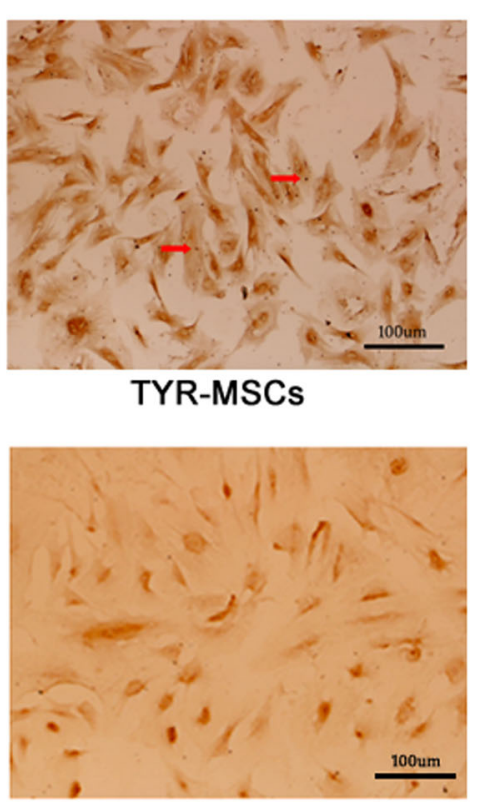

MSCs

Fig. 1 Evaluation of the expression of tyrosinase reporter in vitro. a Photos of the cell pellets from TYR-MSCs and MSCs. $\mathbf{b}$ Western blot assay of tyrosinase expression in TYR-MSCs and MSCs. $\beta$-actin was used as the control. c Masson-Fontana silver staining in TYR-MSCs and MSCs. Scale bar= $100 \mu \mathrm{m}$
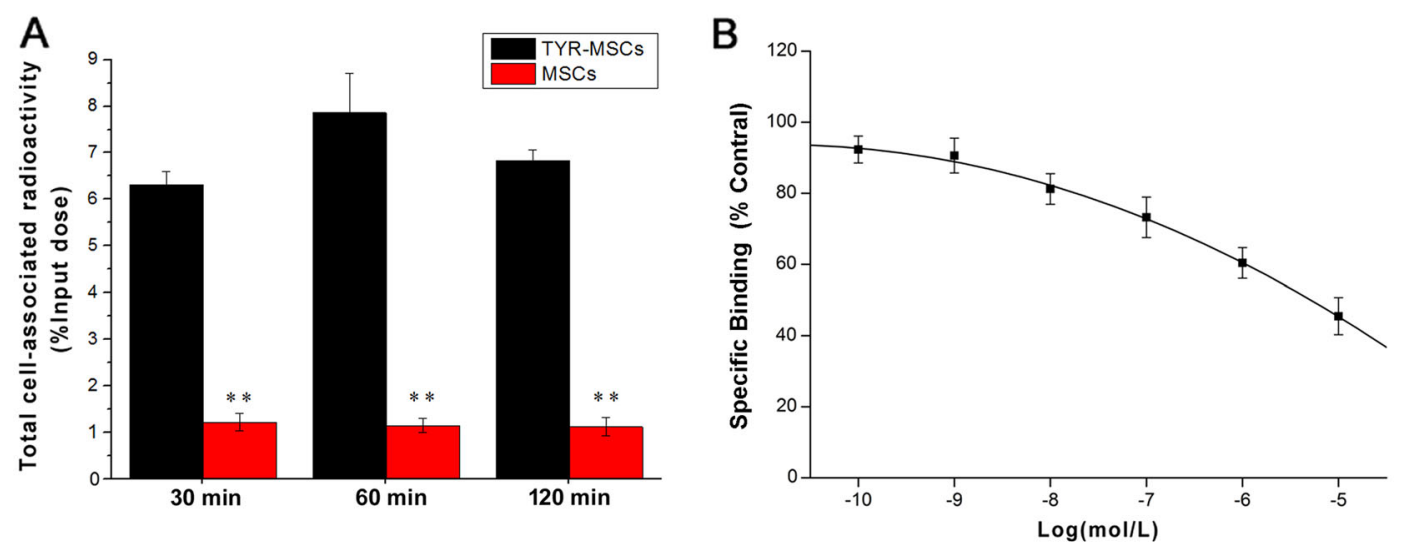

Fig. 2 Cell uptake studies of ${ }^{18} \mathrm{~F}-5$-FPN. a Uptake of ${ }^{18} \mathrm{~F}-5$-FPN in TYR-MSCS and MSCs after incubation with ${ }^{18} \mathrm{~F}-5$-FPN at $37{ }^{\circ} \mathrm{C}$ for 30,60 , and 120 min. ${ }^{* *} P<0.01$. b Competitive cell-binding assay of ${ }^{18} \mathrm{~F}-5$-FPN to TYR-MSCs. The results are expressed as a percentage of cellular uptake inhibition ratio. All results were expressed as a percentage of cellular uptake or binding using the mean of triplicate measurements \pm SD

of $\sim 96 \mathrm{kDa}$ corresponding to tyrosinase (Fig. 1b). MassonFontana staining demonstrated melanin deposition within TYR-MSCs (Fig. 1c). Cellular tyrosinase activity analysis showed that the absorbance at $490 \mathrm{~nm}$ was substantially higher in TYR-MSCs than in MSCs $(0.65 \pm 0.12$ vs $0.11 \pm$ $0.02 ; n=3, P<0.05)$.

\section{Cellular uptake of ${ }^{18} \mathrm{~F}-5$-FPN}

Uptake levels of ${ }^{18}$ F-5-FPN in TYR-MSCs and MSCs at 30, 60, and 120 min are shown in Fig. 2a. The uptake value of TYR-MSCs increased from $6.32 \pm 0.27 \%$ at $30 \mathrm{~min}$ to $7.86 \pm 0.85 \%$ at $60 \mathrm{~min}$, and then slightly decreased to $6.83 \pm 0.24 \%$ at $120 \mathrm{~min}$. The uptake values of MSCs remained at a low level $(1.22 \pm 0.19,1.15 \pm 0.15,1.12 \pm 0.2$ at 30,60 , and $120 \mathrm{~min}$, respectively) and did not show an increase with longer incubation times. The uptake value of TYR-MSCs was significantly higher than those of MSCs at all time points $(P<0.01)$. Administration of cold ${ }^{19} \mathrm{~F}-5$-FPN inhibited the binding of ${ }^{18} \mathrm{~F}-5$-FPN in TYRMSCs in a concentration-dependent pattern as shown in 
Fig. 2b, illustrating the specificity of binding of ${ }^{18}$ F-5-FPN to melanin in vitro.

\section{In vitro MRI and PAI}

T1W1 MRI acquired in different concentrations of cells (Fig. 3a) revealed hyperintensity of the TYR-MSCs. The MR signals increased with increasing cell concentrations. TYR-MSCs treated with both $\mathrm{FeCl}_{3}$ and tyrosine showed a higher signal than cells treated with $\mathrm{FeCl}_{3}$ or tyrosine alone. MSCs produced a low signal on T1WI at all cell concentrations. The signals of MSCs treated with $\mathrm{FeCl}_{3}$ slightly increased with an increasing number of MSCs. Fig. 3 b shows quantitative analysis of MR signals in vitro in all eight groups.

As shown in Fig. 3c, the PAI signals of TYR-MSCs and TYR-MSCs treated with tyrosine increased with increasing cell concentrations. No photoacoustic signals were detected in the MSCs and MSCs treated with tyrosine, even at $5 \times 10^{5}$ cells. Fig. $3 \mathrm{~d}$ shows quantitative analysis of the photoacoustic signals in vitro in TYR-MSCs with and without tyrosine, which shows that tyrosine enhanced the PAI signal.

\section{Histological verification of myocardial infarction}

The percent infarction of the left ventricular mass determined by TTC staining was $38.1 \% \pm 7.3 \%(n=3)$ in AMI animals, whereas normal rats and control models showed no area of infarction (Fig. 4a). Obvious absent uptake of ${ }^{18} \mathrm{~F}$-FDG in the anterior wall was noted on myocardial metabolic imaging of the AMI animals (Fig. 4b), whereas uniform uptake in the myocardium was seen in normal rats and control models. Fig. 4c shows hematoxylin and eosin staining of infarcted myocardium, normal myocardium, and the border between them. Normal myocardial fibers are arranged in neat rows with abundant capillaries. In the AMI models, the

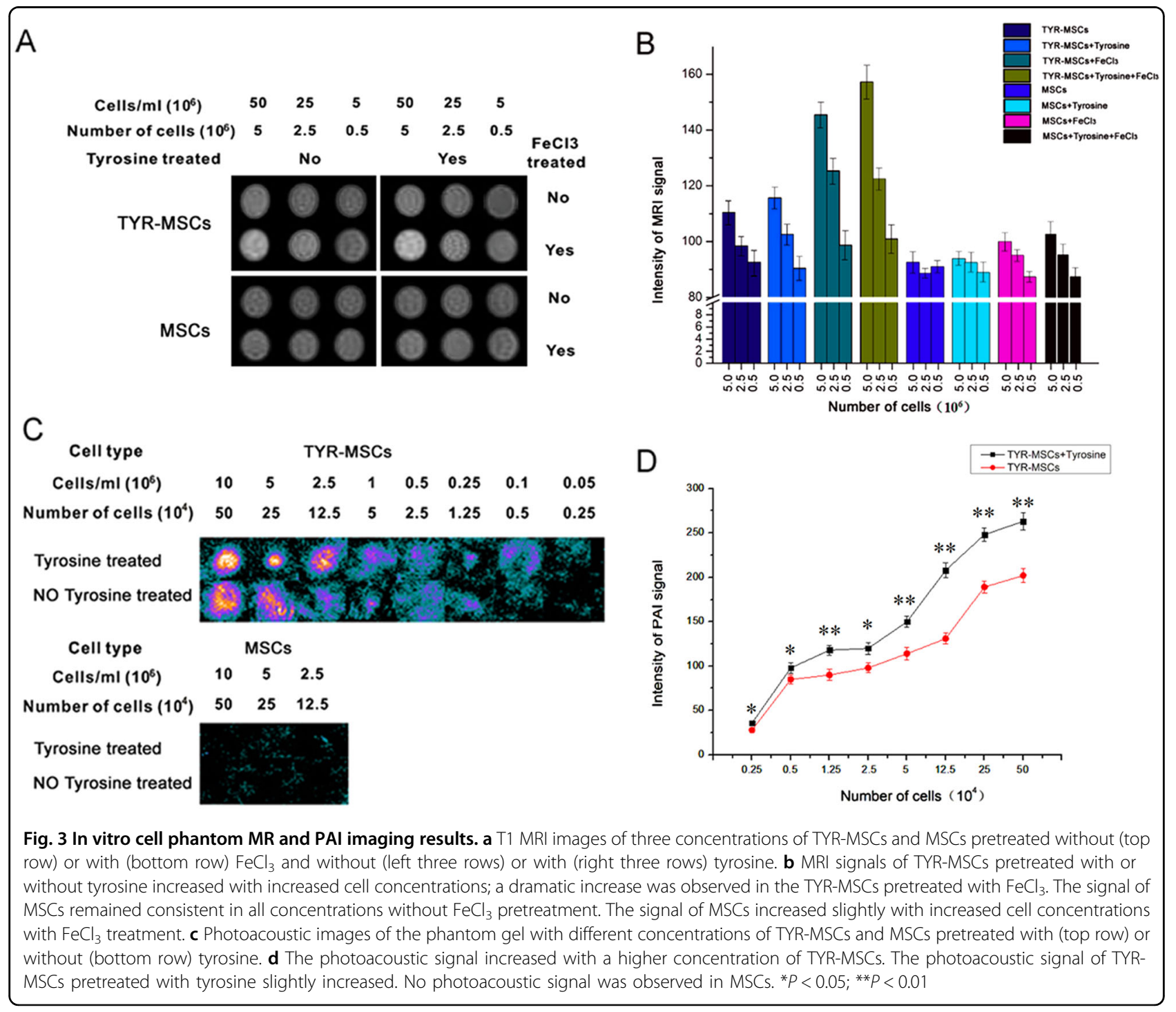



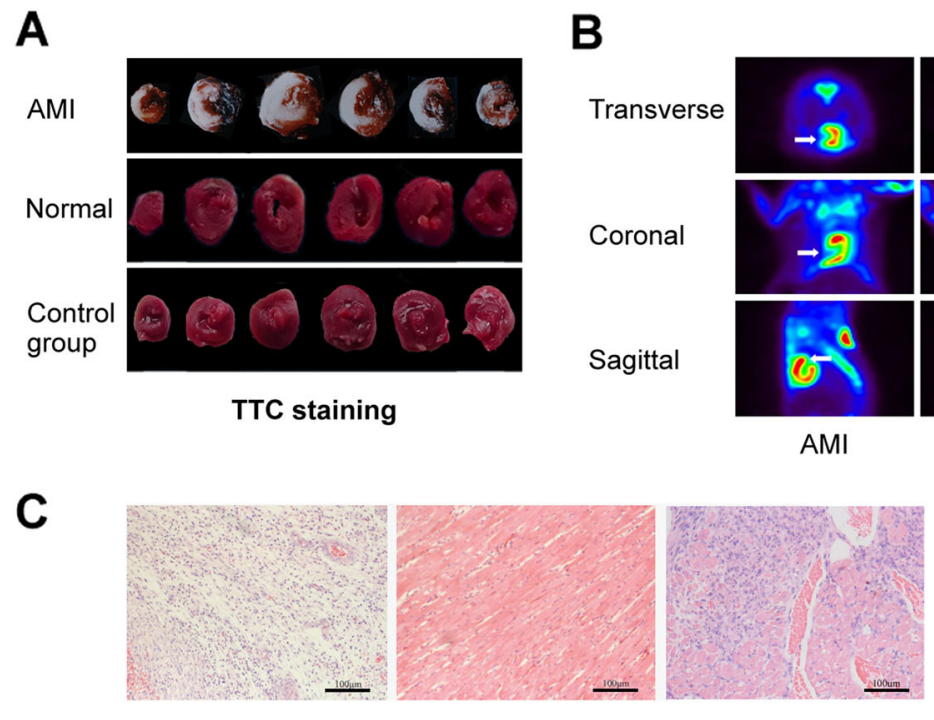

Hematoxylin and eosin staining
B

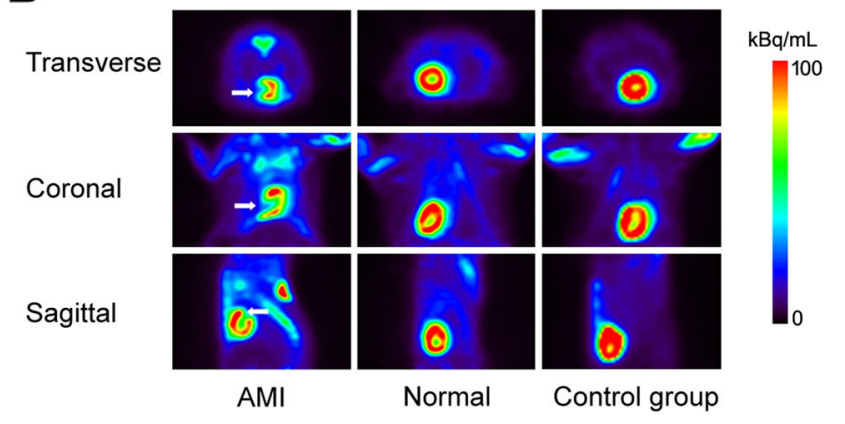

Fig. 4 Verification of myocardial infarction in rat models. a TTC staining of SD rat acute myocardial infarct models (top row), normal SD rats (center row), and control models (bottom row). b Representative decay-corrected transverse (top), coronal (center), and sagittal (bottom) small animal PET images of AMI models (left row), normal rats (center row), and control models (right row) acquired at $1 \mathrm{~h}$ after tail vein injection of ${ }^{18} \mathrm{~F}-$ FDG. The white arrow with no FDG uptake shows the infarct area. However, uniform uptake throughout the heart could be seen in the normal and control group. c HE staining of infarct myocardium (left), normal myocardium (center), and infarction border area (right). Scale bar $=100 \mu \mathrm{m}$

myocardiocytes are necrotic with surrounding edema and denaturation, distinct lymphocyte infiltration, myocardial fiber rupture, and vacuolar denaturation (Fig. 4c).

\section{In vivo PET, MRI, and PAI}

PET images of rats injected with TYR-MSCs and MSCs are shown in Fig. 5; the images were obtained $1 \mathrm{~h}$ after injection of ${ }^{18} \mathrm{~F}-5$-FPN. Obvious uptake of ${ }^{18} \mathrm{~F}-5$-FPN in the transplanted area was seen in the rats with transplanted TYR-MSCs. The signals showed a decreasing trend from $1.78 \pm 0.22 \%$ ID per g to $1.62 \pm 0.13 \%$ ID per $g$ $(n=8)$ from day 1 to 28 post injection but had no significant difference. No ${ }^{18}$ F-5-FPN uptake was seen in the MSCs group and control group.

On the first day after TYR-MSC transplantation, lowintensity MRI signals were visible in the transplanted area on $\mathrm{T}^{*}$ sequences (Fig. 6a). The area of low-intensity signals decreased over time. Signal contrast (\%) of TYRMSCs showed a tendency to increase gradually from $59.18 \pm 4.9$ to $80.5 \pm 8.8(n=8)$, indicating that the intensity of the MRI signal decreased with time. At 28 days after transplantation, the low-intensity signals became unclear. For the MSCs group and control group, no low-intensity signals were observed.

As shown in Fig. 6b, rats transplanted with TYR-MSCs produced high photoacoustic signal intensities, whereas the MSCs group and control group did not produce photoacoustic signals. The PAI signals of TYR-MSCs were $472.6 \pm 48.1,434.8 \pm 51.2,387.3 \pm 62.0,345.9 \pm 79.8$, and $283.4 \pm 49.2$ at $1,7,14,21$, and 28 days post injection, respectively $(n=8)$.

\section{Quantitative analysis of multimodality in vivo}

Fig. 7 shows the quantitative analysis of multimodality in vivo. The data indicated that TYR-MSCs demonstrated clear signals in PET (Fig. 7a), MRI (Fig. 7b), and PAI (Fig. 7c). The signal intensity of MRI and PAI decreased significantly with time $(P<0.05)$; however, there was no significant difference between the PET signals on day 1 and day 28 , which implied that PET could be used to track TYR-MSCs in vivo for longer time than MRI or PAI.

\section{Discussion}

The rapid growth of regenerative medicine has made cell replacement therapy possible for myocardial infarction $^{19-21}$. However, numerous challenges remain in using this therapy, including tracking the location, survival, distribution, and differentiation process of transplanted cells in vivo. Multimodality molecular imaging of reporter genes is a method that allows noninvasive assessment of stem cell therapy and has the potential to diminish the shortcomings of any single imaging modality to gain more complete image ${ }^{22}$.

In this study, stable MSCs expressing melanin were successfully established after lentiviral transfection with the tyrosinase reporter gene. In vitro experiments showed that TYR-MSCs exhibited clear MRI and PAI signals and could specifically take up ${ }^{18}$ F-5-FPN. After injection of 


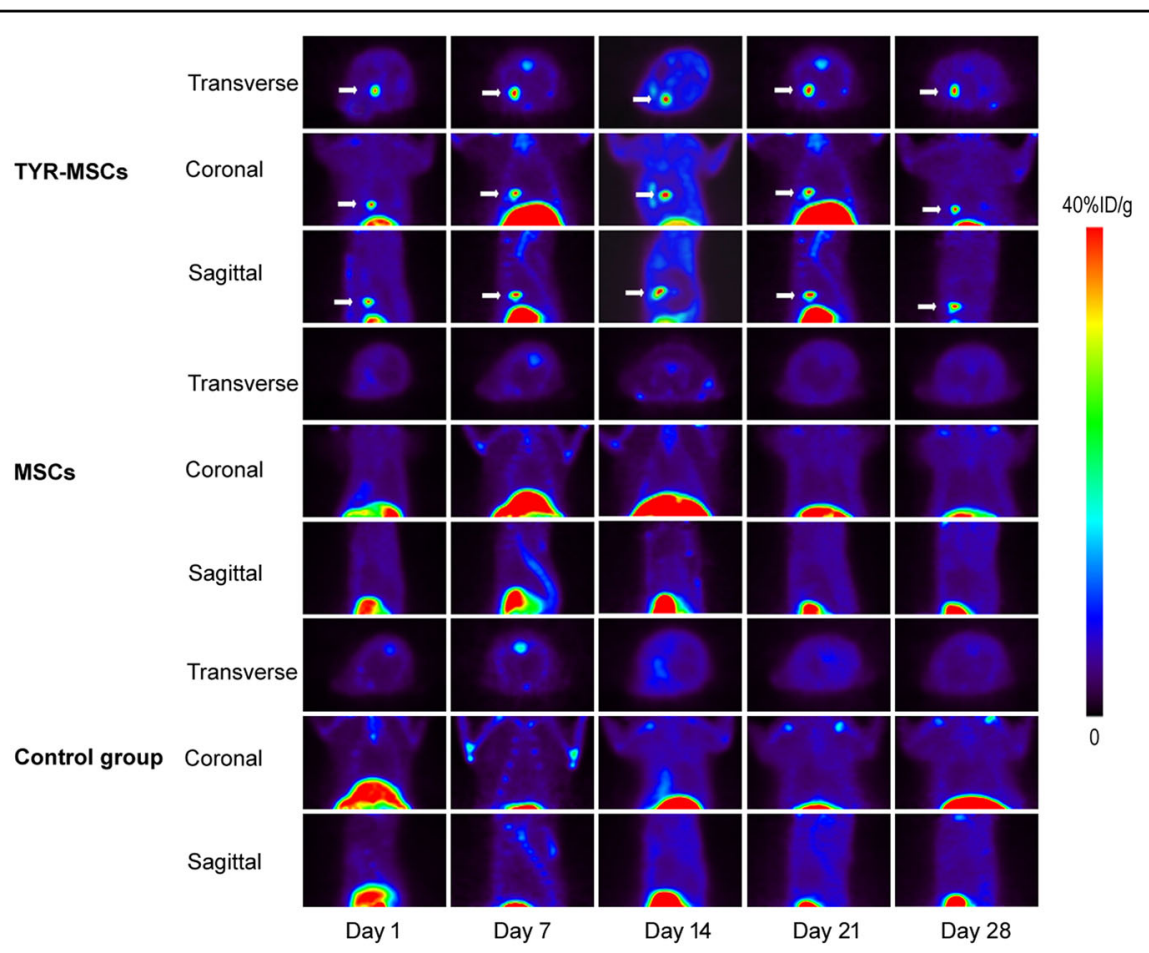

Fig. 5 In vivo ${ }^{18}$ F-5-FPN animal PET imaging. Representative decay-corrected transverse, coronal, and sagittal small animal PET images of TYR-MSC rat models (top three rows) and MSC rat models (center three rows) and control models (bottom three rows) acquired at $1 \mathrm{~h}$ after tail vein injection of ${ }^{18} \mathrm{~F}-5-\mathrm{FPN}$ at $\mathrm{d} 1,7,14,21$, and 28 after transplantation of TYR-MSCS or MSCS

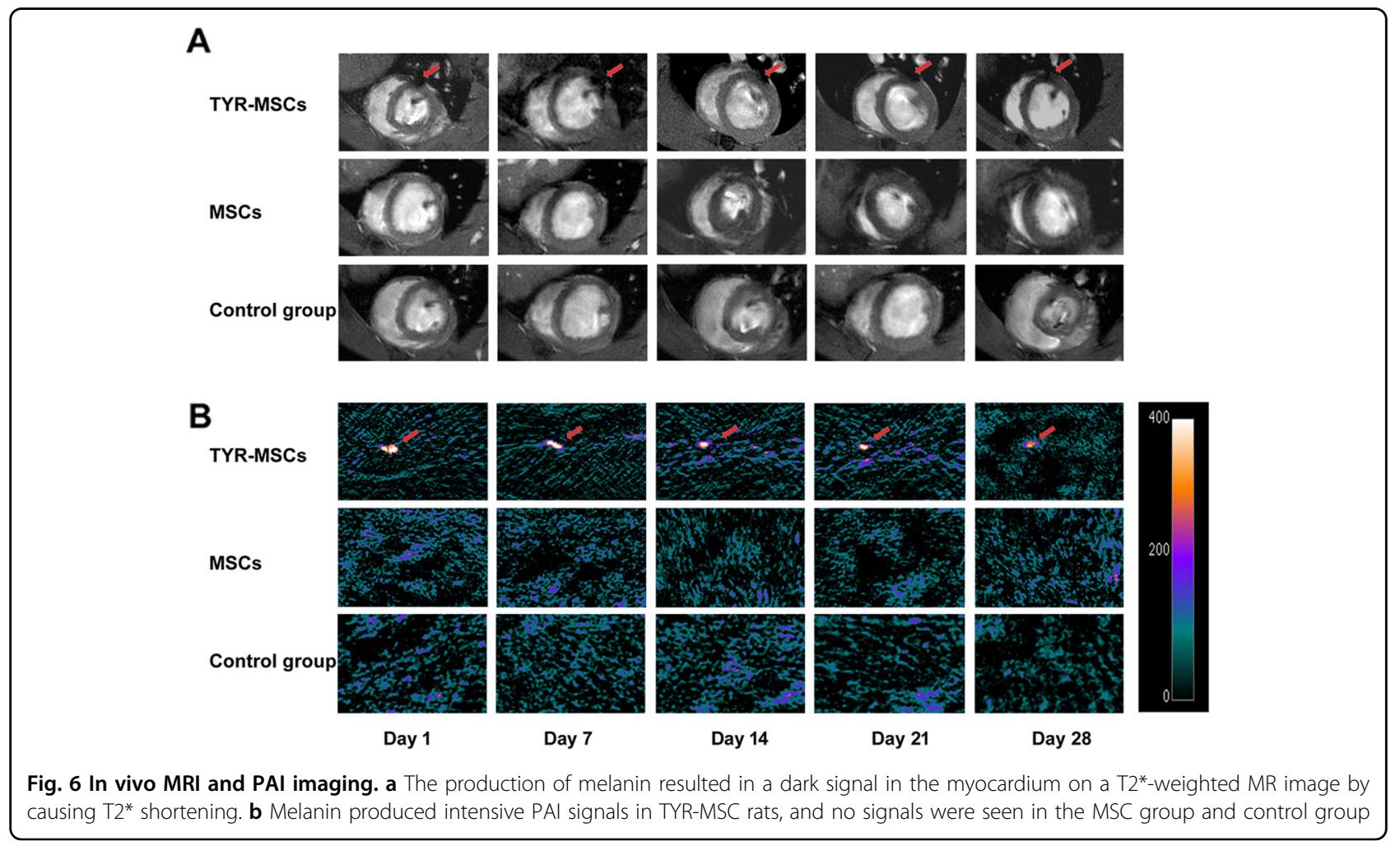



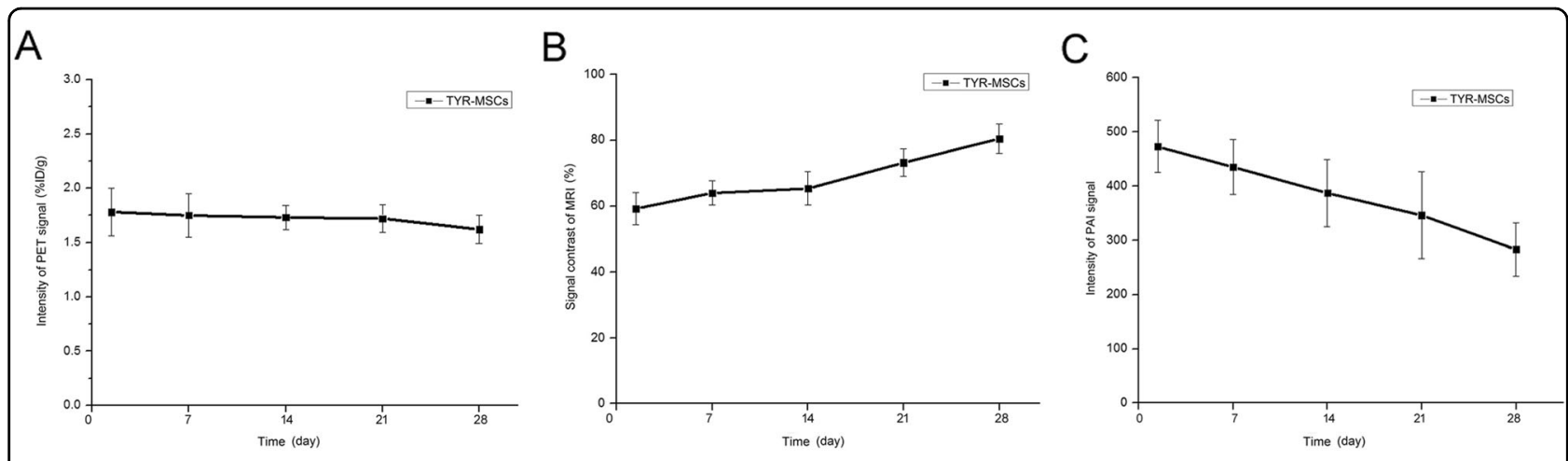

Fig. 7 Semi-quantitative analysis of in vivo multimodality imaging. a The TYR-MSC uptake value slightly decreased with time in the PET serial images. Data are expressed as the percentage administered activity (injected dose) per gram of tissue (\%ID per g). b MRI signal contrast (\%) of TYRMSCs on T2* sequences showed a tendency to increase significantly. c The intensity of the PAl signal decreased obviously with time. Data are expressed as the mean $\pm \mathrm{SD}$

TYR-MSCs into the infarcted myocardium, clear signals could be seen on PET, MRI, and PAI at 28 days, suggesting the stability of melanin in viable TYR-MSCs. To the best of our knowledge, this study is the first time that a stand-alone reporter gene tyrosinase has been used in multimodality monitoring of the fate of stem cells in vivo after transplantation into infarcted myocardium. Tyrosinase, when used as a multimodality reporter gene, does not require co-administration of an enzymatic substrate, and in theory, it should produce plenty of melanin to successfully perform PET, MRI, and PAI if a minimum threshold cell number is reached. Previous studies have indicated that tyrosinase expression exhibits low-level toxicity in mammalian cells ${ }^{23}$, and tyrosinase elicits less of an immune response than other exogenous reporter genes.

The mechanism of tri-modality imaging of the tyrosinase reporter gene has been previously discussed ${ }^{4}$. Although the use of tyrosinase as a tri- or bi-modality reporter gene has been validated previously by us ${ }^{3,4,6}$ and other investigators ${ }^{13}$, the previous approaches had some limitations, such as the inability to obtain stable transduced cell lines, lack of in vivo imaging, or not using PET. Thanks to the newly synthesized probe ${ }^{18} \mathrm{~F}-5$-FPN, which can bind to melanin quickly with high binding capacity and high specificity, tyrosinase gene expression can be detected in vitro and in vivo through ${ }^{18} \mathrm{~F}-5$-FPN by specifically combining with melanin. Based on the technology of lentivirus transfection, stable cell lines were established, and the signals may last for at least 28 days.

PAI is an emerging hybrid molecular imaging tool owing to its unique property of combining optical and acoustic imaging with higher spatial resolution ${ }^{24}$. Melanin has strong optical absorption over a broad spectrum ${ }^{25}$, which allows for good tissue penetration. Meanwhile, high resolution can be maintained in PAI because the photoacoustic wave has low scattering in tissue ${ }^{26}$. Recently, some studies have shown that tyrosinase can be used as a reporter gene for PAI given that tyrosinase modulates melanin synthesis ${ }^{27}$. Märk et al. ${ }^{28}$ transfected rat MSCs with reporter genes co-expressing tyrosinase and a fluorescent protein (mCherry) and performed photoacoustic imaging in small animal models of tissue regeneration. However, their study lacked quantitative analysis of photoacoustic signals and continuous observation in vivo. In our study, $2 \times 10^{6}$ TYR-MSCs cells implanted into the infarcted myocardium produced a strong signal on PAI, suggesting its high sensitivity. In vitro, $2.5 \times 10^{3}$ cells $\left(5 \times 10^{4}\right.$ cells per $\left.\mathrm{mL}\right)$ were sufficient for imaging. Although the intensity of the PAI signal decreased over time, the signal was still visible on $\mathrm{d} 28$, which means PAI is a feasible method for long-term tracking of stem cells in vivo. However, the tissue penetration depth is limited to $5 \mathrm{~cm}$, increasing the difficulty of finding a lesion in deep tissue ${ }^{29}$. Blood flow can also produce strong photoacoustic signals, which may distort the signals produced by TYR-MSCs. PET and MRI could be used to rectify the limitations of PAI.

Among the most important properties of melanin is its ability to strongly chelate metal ions, such as $\mathrm{Cu}^{2+}, \mathrm{Mn}^{2+}$, and $\mathrm{Fe}^{3+}$, leading to shortened $\mathrm{T} 1$ and $\mathrm{T} 2$ relaxation times in vitro or in vivo on $\mathrm{MRI}^{30}$. The typical contrast pattern observable in melanoma shows hyperintensity on T1WI and hypointensity on T2* weighted images. Some studies have reported that microscopic particles of iron oxide bound to MSCs can be tracked in vivo using $\mathrm{MRI}^{31}, 32$. However, the reliability of iron particle tracking of transplanted stem cells has been challenged by several studies because iron particles may be engulfed by macrophages after stem cell death ${ }^{33}$. Microscopic particles of iron oxide also cannot be reproduced by transplanted MSCs, which will decrease the signal duration. Amsalem et al. ${ }^{34}$ labeled rat MSCs with superparamagnetic iron 
oxide nanoparticles to track MSCs in vivo using MRI. After 4-week follow-up, co-staining for iron and ED1 (resident macrophage marker) showed that the ironpositive cells were cardiac macrophages. When nanoparticles were used to track stem cells, cells have to be labeled in vitro, and the signal would be diluted if cells continue to divide, leading to decreased signal intensity per cell. A stable cell line expressing tyrosinase as a reporter gene has better biocompatibility and longer imaging time than cells using microscopic particles or nanoparticles.

Recent studies have demonstrated the feasibility of PET for analyzing the fate of stem cells transplanted into the myocardium in vivo. Kang et al. ${ }^{35}$ labeled stem cells with ${ }^{18} \mathrm{~F}$-FDG and injected them into patients with myocardial infarction via an intracoronary catheter after stenting of infarct-related arteries. PET/computed tomography was performed to trace the injected stem cells. However, the process of labeling was completed in vitro, and the longest tracking time lasted only $20 \mathrm{~h}$ after transplantation. In our previous study, ${ }^{18} \mathrm{~F}-5$-FPN, a benzamide analog specifically targeting melanin in vitro and in vivo with high affinity and retention, was developed. In this study, ${ }^{18} \mathrm{~F}-5$ FPN showed an excellent ability to track TYR-MSCs in vivo. As normal myocardial cells did not take up ${ }^{18} \mathrm{~F}-5$ FPN, myocardial background radiation was very low, which led to the benefit of excellent PET imaging quality. The intensity of PET signals did not show an obvious decrease with time.

Comparing the three imaging modalities, PET imaging showed clear and obvious signals until day 28, which illustrates that the stem cells were alive and produced melanin constantly. MRI signals could also be seen, but they were not as clear as PET signals, and they decreased significantly with time. PAI combines strong optical contrast and high ultrasonic resolution in a single modality, but it provides less anatomical information than MRI. Any single imaging method has some limitations. New multimodality imaging techniques have achieved great progress in the field of diagnostic imaging.

MSCs stably transduced with the tyrosinase gene produce melanin, which is the basis for multimodality imaging with PET, MRI, and PAI for assessing the viability, location, and dwell time of transplanted stem cells. Functional imaging using these cells is a future goal. Thus far, we have performed only transplanted stem cell tracking in the infarcted myocardium. Future work may incorporate quantitative analysis of tyrosinase expression in vivo and the recovery of cardiac function after stem cell transplantation. Most studies on stem cell tracking have focused on the location and duration of stem cell viability. Truly functional multimodality molecular imaging based on reporter gene technology has a promising future.

\section{Limitations}

Some limitations should be mentioned. First, although an AMI model with homogeneous infarct area was established, the results might not be representative of human MI. The location, area, and complications of human MI are highly variable. Multimodality imaging using a tyrosinase reporter gene for assessing transplanted stem cells in human MI may still have a long way to go. Second, permanent coronary artery ligation was performed for the MI model in this study, whereas ischemia/ reperfusion models are more representative and more closely resemble the physiology of MI in human than permanent ligation. Last but not the least, it will be much better to have a known marker (e.g., green fluorescent protein) to validate the viability of tyrosinase, which will also be helpful to verify the specific uptake of ${ }^{18} \mathrm{~F}$-5-FPN in vitro and in vivo. Despite these limitations, we confirmed that MSCs stably transduced with the tyrosinase gene produce melanin, which is the basis for multimodality imaging with PET, MRI, and PAI for assessing the viability, location, and dwell time of transplanted stem cells.

\section{Acknowledgements \\ This work was supported by the National Natural Science Foundation of China (nos. 81371626 and 81630049) and the Clinical Research Physician Program of Tongji Medical College, Huazhong University of Science and Technology (no. 5001530008).}

Conflict of interest

The authors declare that they have no conflict of interest.

\section{Publisher's note}

Springer Nature remains neutral with regard to jurisdictional claims in published maps and institutional affiliations.

Received: 28 August 2017 Revised: 21 February 2018 Accepted: 26 February 2018.

Published online: 27 April 2018

\footnotetext{
References

1. Kim, M. H. et al. Evaluation of safety and efficacy of adipose-derived stem cells in rat myocardial infarction model using hexadecyl-4-[24|] iodobenzoate for cell tracking. Appl. Radiat. Isot. 108, 116-123 (2016).

2. Pei, Z. et al. A multimodality reporter gene for monitoring transplanted stem cells. Nucl. Med. Biol. 39, 813-820 (2012).

3. Qin, C. et al. Tyrosinase as a multifunctional reporter gene for photoacoustic/ MRI/PET triple modality molecular imaging. Sci. Rep. 3, 1490 (2013).

4. Feng, $H$. et al. TYR as a multifunctional reporter gene regulated by the Tet-on system for multimodality imaging: an in vitro study. Sci. Rep. 5, 15502 (2015).

5. Wang, L. V. Multiscale photoacoustic microscopy and computed tomography. Nat. Photonics 3, 503-509 (2009).

6. Feng, $\mathrm{H}$. et al. Imaging malignant melanoma with ${ }^{18} \mathrm{~F}-5-\mathrm{FPN}$. Eur. J. Nucl. Med. Mol. Imaging 43, 113-122 (2016).

7. Fan, Q. et al. Transferring biomarker into molecular probe: melanin nanoparticle as a naturally active platform for multimodality imaging. J. Am. Chem. Soc. 136, 15185-15194 (2014).

8. Zhang, $X$. Y. et al. Lentiviral vectors for sustained transgene expression in human bone marrow-derived stromal cells. Mol. Ther. 5, 555-565 (2002).
} 
9. Annis, D. S. et al. Absence of vitamin K-dependent $\gamma$-carboxylation in human periostin extracted from fibrotic lung or secreted from a cell line engineered to optimize $\gamma$-carboxylation. PLOS ONE 10, e0135374 (2015).

10. DiVito, K. A., Trabosh, V. A., Chen, Y. S., Simbulan-Rosenthal, C. M. \& Rosenthal, D. S. Inhibitor of differentiation-4 (Id4) stimulates pigmentation in melanoma leading to histiocyte infiltration. Exp. Dermatol. 24, 101-107 (2015).

11. Liao, N. et al. Poly (dopamine) coated superparamagnetic iron oxide nanocluster for noninvasive labeling, tracking, and targeted delivery of adipose tissue-derived stem cells. Sci. Rep. 6, 18746 (2016).

12. Enochs, W. S., Petherick, P., Bogdanova, A., Mohr, U. \& Weissleder, R. Paramagnetic metal scavenging by melanin: MR imaging. Radiology 204, 417-423 (1997).

13. Paproski, R. J., Forbrich, A. E., Wachowicz, K., Hitt, M. M. \& Zemp, R. J. Tyrosinase as a dual reporter gene for both photoacoustic and magnetic resonance imaging. Biomed. Opt. Express 2, 771-780 (2011).

14. Takimoto, Y. et al. Augmented expression of neuronal nitric oxide synthase in the atria parasympathetically decreases heart rate during acute myocardial infarction in rats. Circulation 105, 490-496 (2002).

15. Doyle, B. et al. Dynamic tracking during intracoronary injection of 18F-FDGlabeled progenitor cell therapy for acute myocardial infarction. J. Nucl. Med. $\mathbf{4 8}$, 1708-1714 (2007).

16. Long, Q. et al. MRI tracking of bone marrow mesenchymal stem cells labeled with ultra-small superparamagnetic iron oxide nanoparticles in a rat model of temporal lobe epilepsy. Neurosci. Lett. 606, 30-35 (2015).

17. Krauss, J. M. \& Puliafito, C. A. Lasers in ophthalmology. Lasers Surg. Med. 17, 102-159 (1995).

18. Wang, C. et al. RGD-conjugated silica-coated gold nanorods on the surface of carbon nanotubes for targeted photoacoustic imaging of gastric cancer. Nanoscale Res. Lett. 9, 1-10 (2014).

19. Segers, V. F. \& Lee, R. T. Stem-cell therapy for cardiac disease. Nature 451, 937-942 (2008).

20. Stamm, C. et al. Autologous bone-marrow stem-cell transplantation for myocardial regeneration. Lancet 361, 45-46 (2003).

21. Strauer, B. E. \& Kornowski, R. Stem cell therapy in perspective. Circulation 107, 929-934 (2003).

22. Ray, P. Multimodality molecular imaging of disease progression in living subjects. J. Biosci. 36, 499-504 (2011).
23. Weissleder, R. et al. MR imaging and scintigraphy of gene expression through melanin induction. Radiology 204, 425-429 (1997).

24. Mallidi, S., Luke, G. P. \& Emelianov, S. Photoacoustic imaging in cancer detection, diagnosis, and treatment guidance. Trends Biotechnol. 29, 213-221 (2011).

25. Viator, J. A. et al. A comparative study of photoacoustic and reflectance methods for determination of epidermal melanin content. J. Invest. Dermatol. 122, 1432-1439 (2004).

26. Xu, M. \& Wang, L. V. Photoacoustic imaging in biomedicine. Rev. Sci. Instrum. 77, 041101 (2006).

27. Krumholz, A. et al. Photoacoustic microscopy of tyrosinase reporter gene in vivo. J. Biomed. Opt. 16, 080503 (2011).

28. Märk, J. et al. Development of tyrosinase-based reporter genes for preclinical photoacoustic imaging of mesenchymal stem cells. In Spie Bios $\mathbf{8 9 4 3}, 89433 Z$ (2014).

29. Kothapalli, S. R. et al. Deep tissue photoacoustic imaging using a miniaturized 2-D capacitive micromachined ultrasonic transducer array. IEEE Trans. Biomed. Eng. 59, 1199-1204 (2012).

30. Ju, K. Y. et al. Bio-inspired, melanin-like nanoparticles as a highly efficient contrast agent for T1-weighted magnetic resonance imaging. Biomacromolecules 14, 3491-3497 (2013).

31. Drey, F. et al. Noninvasive in vivo tracking of mesenchymal stem cells and evaluation of cell therapeutic effects in a murine model using a clinical $3.0 \mathrm{~T}$ MRI. Cell Transplant. 22, 1971-1980 (2013).

32. Boulland, J. L. et al. Evaluation of intracellular labeling with micron-sized particles of iron oxide (MPIOS) as a general tool for in vitro and in vivo tracking of human stem and progenitor cells. Cell Transplant. 21, 1743-1759 (2012).

33. Chen, $\mathrm{X}$. et al. Dynamic tracking of injected mesenchymal stem cells after myocardial infarction in rats: a serial $7 T$ MRI study. Stem Cells Int. 2016 4656539 (2016).

34. Amsalem, Y. et al. Iron-oxide labeling and outcome of transplanted mesenchymal stem cells in the infarcted myocardium. Circulation 116, 138-145 (2007).

35. Kang, W. J. et al. Tissue distribution of 18F-FDG-labeled peripheral hematopoietic stem cells after intracoronary administration in patients with myocardial infarction. J. Nucl. Med. 47, 1295-1301 (2006). 